\title{
Observation of polychromatic vortex solitons
}

\author{
Dragomir N. Neshev ${ }^{1}$, Alexander Dreischuh ${ }^{1,2}$, Vladlen Shvedov ${ }^{1,3}$, \\ Anton S. Desyatnikov ${ }^{1}$, Wieslaw Krolikowski ${ }^{1}$, and Yuri S. Kivshar ${ }^{1}$ \\ ${ }^{1}$ Nonlinear Physics Center and Laser Physics Center, Research School of Physical Sciences \\ and Engineering, \\ Australian National University, Canberra ACT 0200, Australia \\ 2 Department of Quantum Electronics, Sofia University, Sofia-1164, Bulgaria \\ ${ }^{3}$ Department of Physics, Taurida National University, Simferopol 95007 Crimea, Ukraine
}

\begin{abstract}
We demonstrate experimentally the formation of polychromatic single- and double-charge optical vortex solitons by employing a lithium niobate crystal as a nonlinear medium with defocusing nonlinearity. We study the wavelength dependence of the vortex core localization and observe self-trapping of polychromatic vortex-carrying beams with the bandwidth spanning over more than $70 \mathrm{~nm}$ in wavelength for single-charge and $180 \mathrm{~nm}$ for double-charge vortex solitons. (c) 2008 Optical Society of America
\end{abstract}

OCIS codes: $190.4420,190.6135,260.6042$

The suppression of natural diffraction of light due to nonlinear self-focusing is a fascinating phenomenon that may lead to the formation of spatial optical solitons [1]. In defocusing nonlinear media, both nonlinearity and diffraction contribute to the spreading of finite-size beams. As such, the formation of bright solitons is inhibited, but self-trapping of "dark filaments" can be observed instead [2]. This type of self-trapping leads to the formation of two-dimensional dark solitons with phase singularities, the entities well-known in the context of nonlinear optics and Bose-Einstein condensates [1]. A dark soliton localized in two dimensions inherently carries an optical vortex (OV) - an isolated defect of the wave front with a singularity of its phase and a twisted power flow [3]. While single-wavelength vortex solitons have been routinely observed, the experimental realization of the multi-color vortex solitons remains an experimental challenge. Only two-color simultaneous trapping of parametrically interacting optical waves have been predicted and observed so far $[4,5]$.

Simultaneous trapping of many chromatic components requires their incoherent interaction via cross-phase modulation in a Kerr-type medium [6]. Trapping of multi-frequency components appears similar to the trapping of mutually incoherent modes of partially coherent light but with the important difference that the polychromatic beam is spatially coherent 


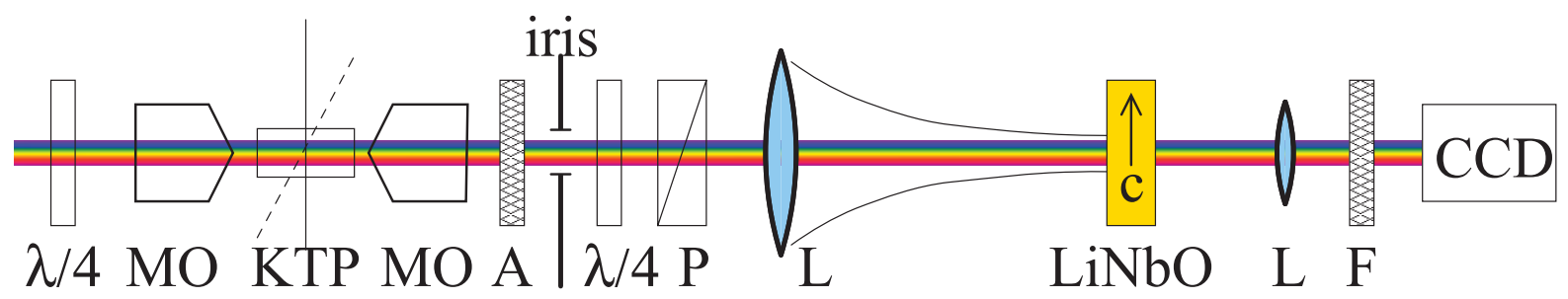

Fig. 1. (color online) Experimental setup: $\lambda / 4$ - quarter waveplate, A - attenuator, MO microscope objectives, SF - spatial filter, L - lenses, P - polarizer, F - filter, KTP - Potassium Titanium Oxide Phosphate (with adjustable tilting), and LiNbO - Lithium Niobate crystals.

and posses a well-defined phase structure. While mutual trapping of many spatially incoherent vortex components at a single wavelength was observed a decade ago [7], the trapping of an optical vortex consisting of many spectral components has never been realized so far. The major obstacle is the chromatic dispersion present in the various methods of vortex generation, leading to spatial separation of individual vortex colors. Novel chromatic effects near the core of a spatially coherent polychromatic vortex were predicted [8] and recently observed for 'white-light' [9] and 'rainbow' [10] vortex beams, as well as for ultra-short pulse vortices [11]. In addition, the spin-orbit coupling in uniaxial crystals has also been successfully implemented to generate white-light partially coherent vortices of the single [12] and double [13] charges.

In this Letter we demonstrate, for the first time to our knowledge, the formation of spatially coherent white-light single- and double-charge optical vortex solitons. These broadband vortex beams are generated in supercontinuum radiation by employing an uniaxial Potassium Titanium Oxide Phosphate (KTP) crystal $[12,13]$ and and they propagate in a Lithium Niobate $\left(\mathrm{LiNbO}_{3}\right)$ crystal used here as a nonlinear medium with defocusing nonlinearity. We show that the defocusing nonlinear response of $\mathrm{LiNbO}_{3}$ leads to mutual trapping of colors in the spectral window of $70 \mathrm{~nm}$ for single-, and $180 \mathrm{~nm}$ for double-charge vortex solitons.

In our experiments we use a supercontinuum light as a coherent white-light source (see Fig. 1). The supercontinuum is generated by $140 \mathrm{fs}$ laser pulses (at $800 \mathrm{~nm}$ ) in a $1 \mathrm{~m}$ long nonlinear photonic-crystal fiber and spans the spectral range $450-700 \mathrm{~nm}$. The linearly polarized polychromatic beam can be converted to a circular polarization by an achromatic $(450-800 \mathrm{~nm}) \lambda / 4$ wave-plate. The beam is then focused by a microscope objective $(\times 10)$ in a $7 \mathrm{~mm}$ long KTP crystal to create desired vortex structure of the input beam. After passing through the crystal the beam is subsequently re-collimated by a second objective $(\times 3)$, attenuated by neutral density filters (A), and spatially filtered by an iris diaphragm. When the optical axis of the uniaxial KTP crystal is oriented parallel to the beam propagation, the beam attains a linearly-polarized double-charged optical vortex structure as described earlier [13]. When the quarter waveplates are removed and with the polarizer oriented par- 
allel to the polarization of the input beam, we generate topological quadrupole from which a single-charged OV is isolated by tilting the optical axis of the KTP crystal [12]. The generated single-charge vortex beam is then focused by an achromatic lens ( $f=120 \mathrm{~mm})$ at the entrance facet of a $15 \mathrm{~mm}$ long $\mathrm{Fe}$-doped $\mathrm{LiNbO}_{3}$ photorefractive crystal. Input polarization of the beam is set to coincide with the optical c-axis of the crystal in order to maximize its nonlinear response. Additional achromatic lens is used to image either the input or the output crystal facet onto a color CCD camera. A variable $(300-750 \mathrm{~nm})$ spectral filter (F) is used in front of the camera in order to distinguish the different spectral components.

The intensity distribution of the $\mathrm{OV}$ at the exit of the nonlinear $\mathrm{LiNbO}_{3}$ crystal, just after the light beam has been turned on (total power $\sim 50 \mu \mathrm{W}$ ), is shown in the left inset in Fig. 2(a). As the nonlinear response of the crystal is slow this corresponds to a purely linear propagation regime. Subsequent images of the polychromatic OVs are recorded in 5 min. intervals in order to measure the core full width at half maximum (FWHM) as a function of time [Fig. 2(a)]. It is evident that the core of the vortex beam exhibits clear localization with time, reaching a steady state after approximately $25 \mathrm{~min}$. This twofold decrease in size, from approximately $36 \mu \mathrm{m}$ down to $18 \mu \mathrm{m}$ (below the size of $24 \mu \mathrm{m}$ at the input) is a strong indication for the formation of a polychromatic optical vortex soliton. On the other hand, the relatively complex coloring of the intensity distribution at the end of the observation period [see Fig. 2(a), right inset] indicates that the different spectral components of the polychromatic vortex experience different nonlinear behavior. This difference is seen in Fig. 2(b) where we compare the linear (solid squares, dashed line) and nonlinear (open circles) OV FWHM for different spectral components.

From this experimental data one can see that only short wavelength components of the polychromatic vortex are actually localized. This means that the polychromatic vortex soliton is generated only in the wavelength range $460 \sim 532 \mathrm{~nm}$. The spatial localization becomes weaker with increasing the wavelength and approaches the linear diffraction size at $622 \mathrm{~nm}$. This difference in the spectral localization is due to two major factors. Firstly, the sensitivity of photorefractive nonlinearity of the $\mathrm{LiNbO}_{3}$ crystal is strongly dependent on the wavelength [14]. The strongest contribution to the nonlinearity comes from the blue spectral components, which also experience weakest diffraction. The red components on the other hand, diffract much stronger and, therefore, they experience weaker localization.

The other and more important reason for a lack of the vortex core localization at long wavelengths is the spatial dispersion of the OV core positions. This difference in spatial positions of the OV spectral components [12] is due to the dispersion of the tilted KTP crystal [12], causing the OV cores at different wavelengths to enter the nonlinear medium with a slight spatial offset. This is seen in Fig. 3(top graph) showing spread of the OV spectral components in the linear regime of 15 and $25 \mu \mathrm{m}$ in $x$ and $y$ direction respectively. 
As seen in the bottom graph of Fig. 3, nonlinearity gradually decreases the OV core spreading, but only along the crystalline c-axis leaving the spreading in the perpendicular direction nearly unaffected. This spreading suppression is somewhat analogous to the selftrapping of bright incoherent vortices in self-focusing media [15], but it is strongly affected by an anisotropic photovoltaic nonlinearity. We note that due to charge diffusion inside the $\mathrm{LiNbO}_{3}$ crystal, the actual vortex positions at the exist in the linear and nonlinear regimes differ by $120 \mu \mathrm{m}$ along the direction of the c-axis.

After having successfully observed the generation of single-charge vortex solitons we study now the formation of higher-charge localized structures, namely double-charge vortex solitons. Importantly, the experimental scheme for generating double-charge polychromatic optical vortex [13] does not require tilting of the KTP crystal and, therefore, all OV spectral components overlap inside the $\mathrm{LiNbO}_{3}$ crystal, in contrast to the case of a single-charge OV. Therefore, for the generation of a double-charge OV the bandwidth is determined solely by the achromaticity of the $\lambda / 4$ wave-plates.

The graph in Fig. 4 shows the FWHM of the double-charge polychromatic OV core at different spectral components. The vortex size is measured for both linear (low input power) and nonlinear (total power $\sim 50 \mu \mathrm{W}$ ) regimes. We observe linear diffraction of the different spectral components (solid squares, dashed line) with the vortex-core size increasing monotonically with the wavelength. Surprisingly, in the nonlinear regime all OV spectral components are mutually trapped and localized at the same size of approximately $122 \mu \mathrm{m}$, at the crystal output. This result demonstrates a successful formation of a polychromatic double-charge OV soliton of more than $180 \mathrm{~nm}$ spectral bandwidth.

It is worth mentioning that when the polychromatic vortex beam is left to illuminate the crystal for more than three hours, which results in stronger nonlinear response of the crystal, the double-charge OV soliton splits in two well-distinguished single-charge vortices due to the intrinsic higher-charge nonlinear vortex instability [16,17].

In conclusion, we have demonstrated that single- and double-charge polychromatic optical vortex solitons can be generated in defocusing photorefractive nonlinear media. We have observed that for single-charge vortices, the nonlinear self-action of the polychromatic vortex beam leads to a significant suppression of the dispersion in positions of the individual vortex spectral components. In the case of double-charge vortices we have achieved the formation of a vortex soliton over a significant spectral bandwidth with mutually trapped spectral components.

This work was supported by the Australian Research Council through Discovery and Linkage projects and by the NSF-Bulgaria through project WUF-02/05. 


\section{References}

1. Yu. S. Kivshar and G. P. Agrawal, Optical Solitons: From Fibers to Photonic Crystals (Academic Press, San Diego, 2003).

2. G. A. Swartzlander, Jr. and C. T. Law, "Optical vortex solitons observed in Kerr nonlinear media," Phys. Rev. Lett. 69, 2503 (1992).

3. A. S. Desyatnikov, Yu. S. Kivshar, and L. Torner, "Optical vortices and vortex solitons," in Progress in Optics, Vol. 47, Ed. E. Wolf (North-Holland, Amsterdam, 2005).

4. T. J. Alexander, A. V. Buryak, and Yu. S. Kivshar, "Stabilization of dark and vortex parametric spatial solitons," Opt. Lett. 23, 670 (1998).

5. P. Di Trapani, W. Chinaglia, S. Minardi, A. Piskarskas, and G. Valiulis, "Observation of quadratic optical vortex solitons," Phys. Rev. Lett. 84, 3843 (2000).

6. M. Mitchell and M. Segev, "Self-trapping of incoherent white light," Nature 387, 880 (1997).

7. Z. Chen, M. Mitchell, M. Segev, T. H. Coskun and D. N. Christodoulides, "Self-trapping of dark incoherent light beams," Science 280, 889 (1998).

8. M. Berry, "Coloured phase singularities," New J. Phys. 4, 66 (2002).

9. J. Leach and M. J. Padgett, "Observation of chromatic effects near a white-light vortex," New J. Phys. 5, 154 (2003).

10. M. S. Soskin, P. V. Polyansky, and O. O. Arkheluyk, "Computer-synthesized hologram-based rainbow vortices," New J. Phys. 6, 196 (2004).

11. K. Bezuhanov, A. Dreischuh, G. G. Paulus, M. G. Schätzel, H. Walther, "Vortices in femtosecond laser fields," Opt. Lett. 29, 1942 (2004).

12. A. Volyar, V. Shvedov, T. Fadeyeva, A. S. Desyatnikov, D. N. Neshev, W. Krolikowski, and Yu. S. Kivshar, "Generation of single-charge optical vortices with an uniaxial crystal," Opt. Express 14, 3724 (2006).

13. V. Shvedov, W. Krolikowski, A. Volyar, D. N. Neshev, A. S. Desyatnikov, and Yu. S. Kivshar, "Focusing and coherence properties of white-light optical vortices," Opt. Express 13, 7393 (2005).

14. D. N. Neshev, A. A. Sukhorukov, A. Dreischuh, R. Fischer, S. Ha, J. Bolger, L. Bui, W. Krolikowski, B. J. Eggleton, A. Mitchell, M. W. Austin, and Yu. S. Kivshar, "Nonlinear spectralspatial control and localization of supercontinuum radiation," Phys. Rev. Lett. 99, 123901 (2007).

15. C. C. Jeng, M. F. Shih, K. Motzek, and Yu. S. Kivshar, "Partially incoherent optical vortices in self-focusing nonlinear media," Phys. Rev. Lett. 92, 043904 (2004).

16. A. V. Mamaev, M. Saffman, And A. A. Zozulya, "Vortex evolution and bound pair formation in anisotropic nonlinear optical media," Phys. Rev. Lett. 77, 4544 (1996).

17. A. Dreischuh, G. G. Paulus, F. Zacher, F. Grasbon, D. Neshev, and H. Walther, "Modulational instability of multiple-charged optical vortex solitons under saturation of the nonlinearity," Phys. Rev. E 60, 7518 (1999). 
(a)

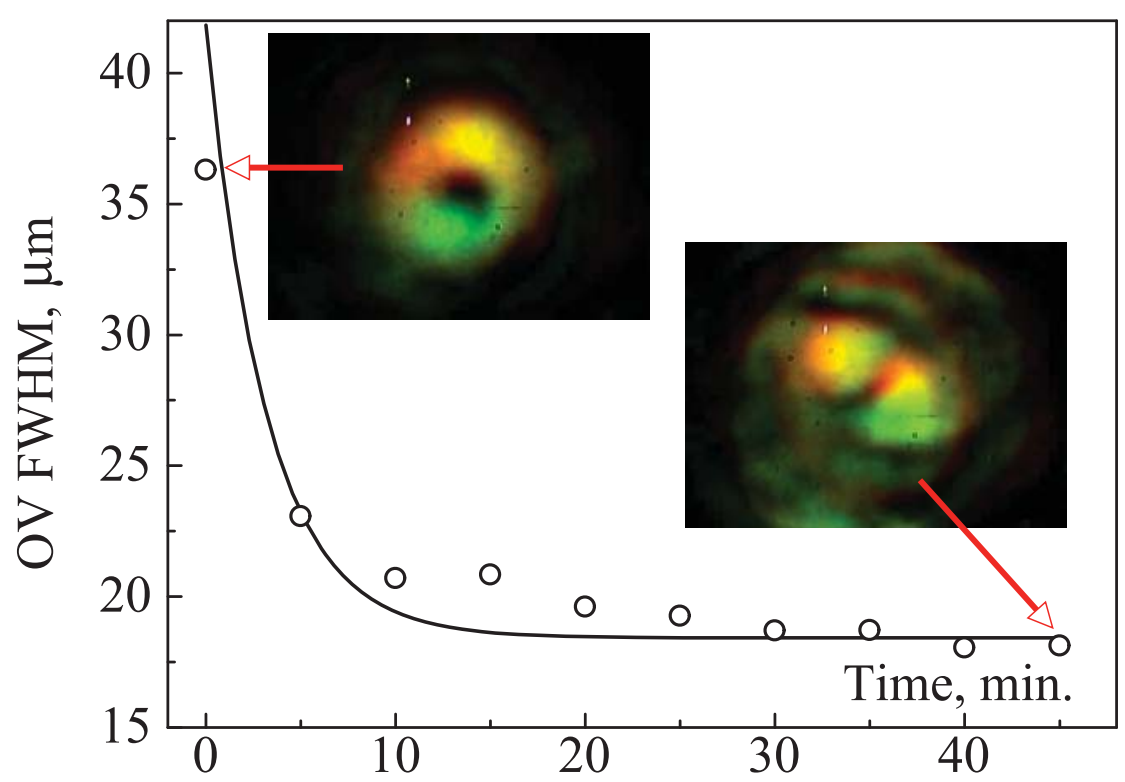

(b)

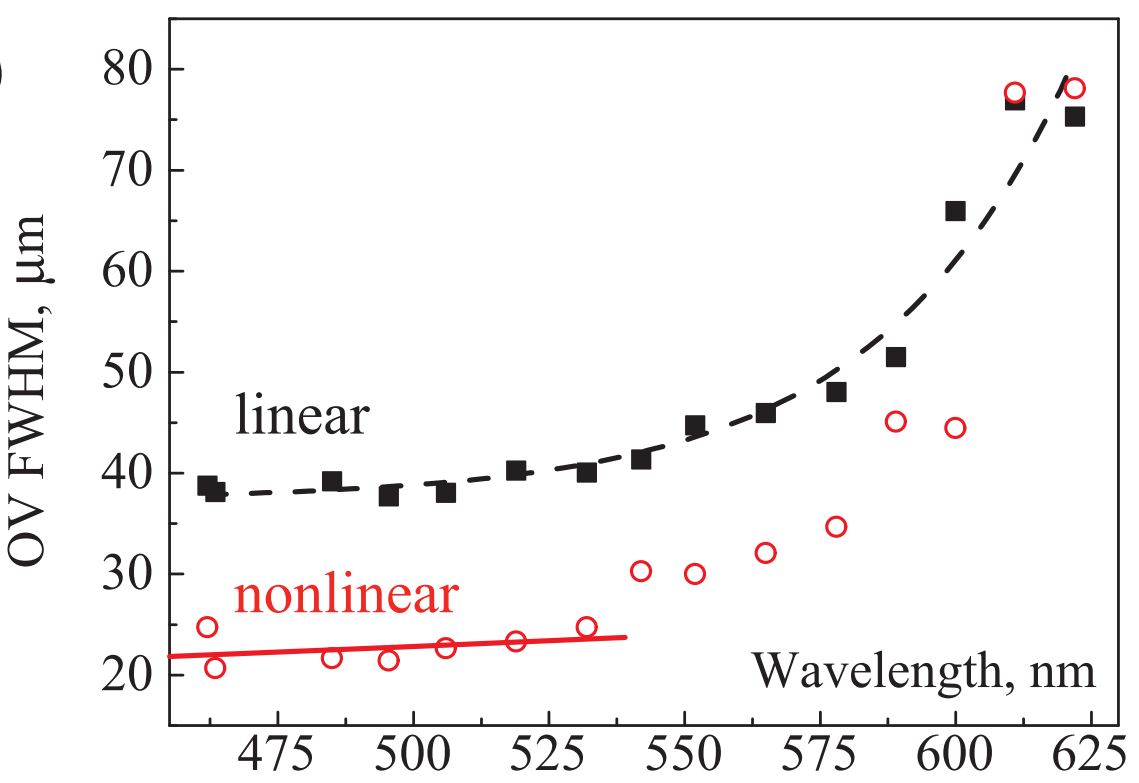

Fig. 2. (color online) (a) Dependence of the average core width of a single-charge polychromatic OV vs. time (input power of $\sim 50 \mu \mathrm{W}$ ). Inset - output vortex intensity distribution in the linear (left) and nonlinear (right) regimes. (b) Width of the OV cores for different spectral components in linear (solid squares; dashed curve) and nonlinear (open circles) regimes. Solid line indicates the wavelength range of the polychromatic OV soliton. 


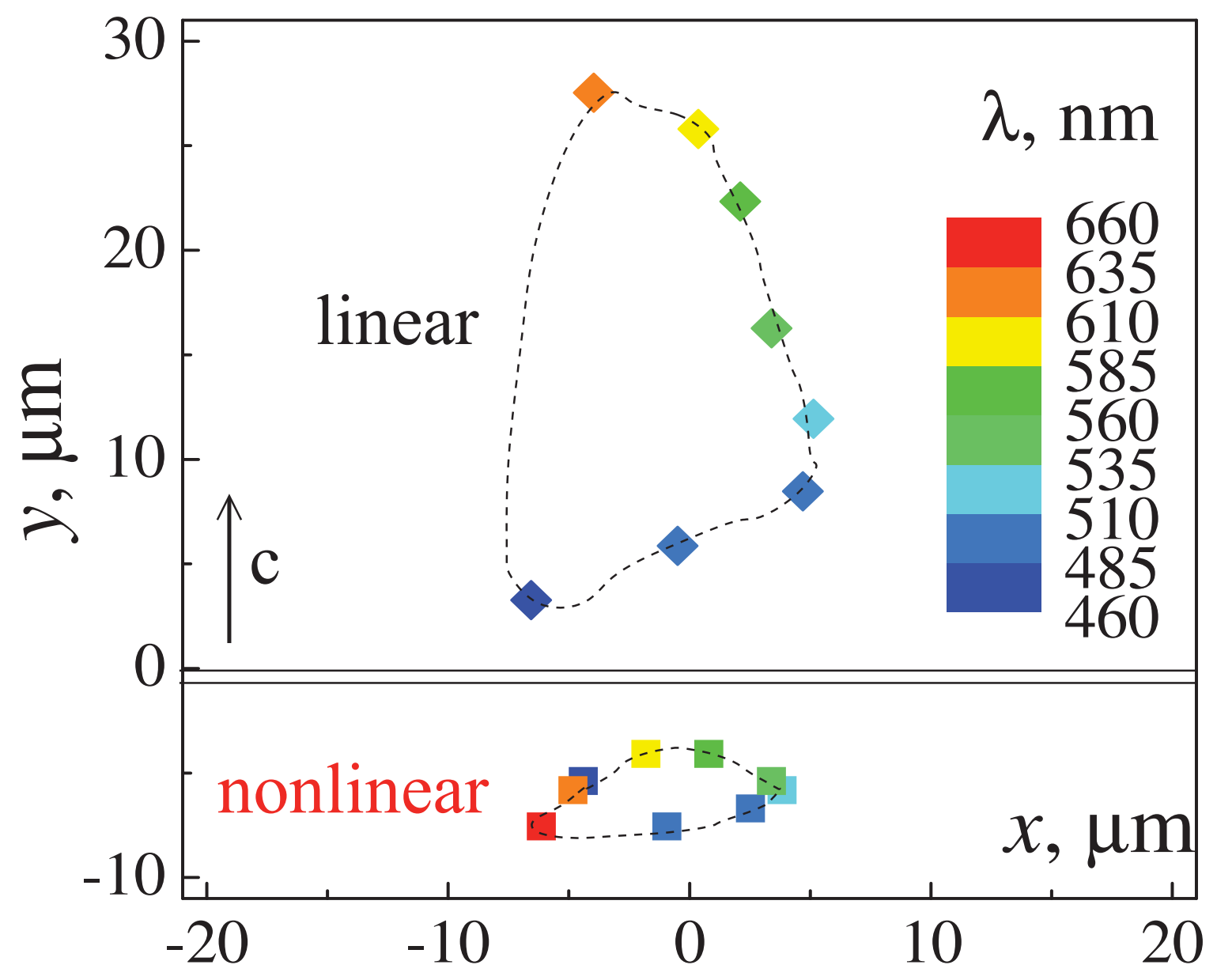

Fig. 3. (color online) (a) Transverse coordinates of the vortex cores at different spectral components in the linear and nonlinear regime. 


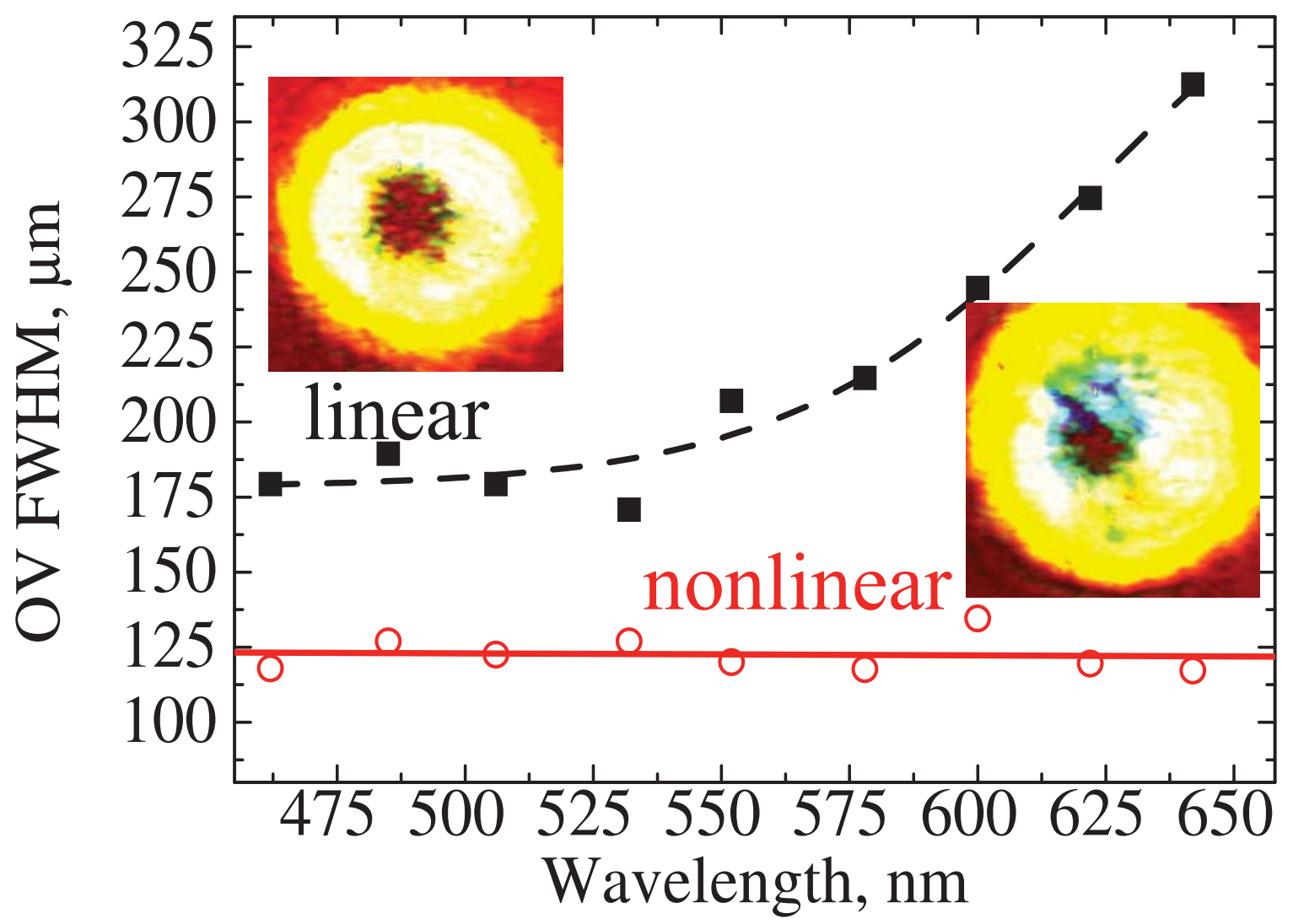

Fig. 4. (Color online) Optical vortex width at individual spectral components in linear regime (solid squares) and nonlinear regime (open circles) of polychromatic double-charged OV soliton formation. Insets - output intensity distributions in the two regimes. 